\section{In Reply: Identification of Women at Heightened Risk for Breast Cancer}

\section{TO THE EDITORS:}

In their letter, Harrissis et al. highlight the important tasks of identifying women at heightened risk for breast cancer and offering risk-appropriate screening and riskreducing surgery. Prospective evidence supports the use of screening magnetic resonance imaging (MRI) for wellselected patients with a documented $B R C A 1 / 2$ gene or a family history suggestive of hereditary breast cancer syndrome. The authors also appropriately question the rising rates of contralateral prophylactic mastectomy (CPM) in the United States identified through the Surveillance, Epidemiology, and End Results Program database as well as several single-institution studies, including our own. ${ }^{1,2}$

The routine use of breast MRI in the preoperative evaluation of women with newly diagnosed breast cancer remains controversial. MRI can detect clinically occult cancers, although outcome studies demonstrating improvements in local control, breast cancer-specific survival, or overall survival are lacking. It is also not clear that preoperative MRI results in higher rates of margin negative resections or lower reoperation rates. ${ }^{3} \mathrm{We}$ acknowledge that the absence of information about the MRI results or subsequent biopsies is a limitation of our study. However, patients who underwent contralateral prophylactic mastectomy did not have a cancer diagnosis in the prophylactic breast. We cannot comment on whether the positive association between MRI and CPM is stronger for those women with false-positive MRI findings than for women with a negative MRI. In our multivariate analysis that controlled for factors associated with decisions for prophylactic surgery, we found that receipt of breast MRI was positively associated with CPM. Additional research is needed to better understand this relationship. Furthermore, because MRI results in additional biopsies and often more extensive surgery, further work on the cost consequences of MRI is warranted.

Melony E. S. Sorbero, PhD, MS, MPH ${ }^{1}$, Andrew W. Dick, PhD ${ }^{1}$, Ellen Burke Beckjord, PhD, MPH ${ }^{1}$, and Gretchen Ahrendt, MD, FACS ${ }^{2}$

${ }^{1}$ RAND Corporation, Pittsburgh, PA;

${ }^{2}$ Magee Womens Hospital, Pittsburgh, PA

e-mail: msorbero@rand.org

Published Online: 9 June 2009

(C) Society of Surgical Oncology 2009

\section{REFERENCES}

1. Tuttle TM, Habermann EB, Grund EH, et al. Increasing use of contralateral prophylactic mastectomy for breast cancer patients: a trend toward more aggressive surgical treatment. J Clin Oncol. 2007;25:5203-9.

2. Sorbero ME, Dick AW, Beckjord EB, Ahrendt G. Diagnostic breast magnetic resonance imaging and contralateral prophylactic mastectomy. Ann Surg Oncol. 2009;16:1597-605.

3. Drew PJ, Harvey I, Hanby A, et al. The UKNIHR Multicentre Randomized COMICE Trial of MRI planning for breast conserving treatment of breast cancer. 31st Annual Meeting of the San Antonio Breast Cancer Symposium, San Antonio, TX, abstract 51; Dec 2008. 